Bull. Korean Math. Soc. 49 (2012), No. 4, pp. 685-692

http://dx.doi.org/10.4134/BKMS.2012.49.4.685

\title{
CLASSES OF HYPERSURFACES WITH VANISHING LAPLACE INVARIANTS
}

\author{
Carlos M. C. Riveros and Armando M. V. Corro
}

\begin{abstract}
Consider a hypersurface $M^{n}$ in $\mathbb{R}^{n+1}$ with $n$ distinct principal curvatures, parametrized by lines of curvature with vanishing Laplace invariants.

(1) If the lines of curvature are planar, then there are no such hypersurfaces for $n \geq 4$, and for $n=3$, they are, up to Möbius transformations, Dupin hypersurfaces with constant Möbius curvature.

(2) If the principal curvatures are given by a sum of functions of separated variables, there are no such hypersurfaces for $n \geq 4$, and for $n=3$, they are, up to Möbius transformations, Dupin hypersurfaces with constant Möbius curvature.
\end{abstract}

\section{Introduction}

A hypersurface $M$ is said to be Dupin if each principal curvature is constant along its corresponding surface of curvature. The Dupin hypersurface $M$ is said to be proper if the number $g$ of distinct principal curvatures is constant on $M$. The class of Dupin hypersurfaces is invariant under Lie transformations [9]. Therefore, the classification of Dupin hypersurfaces is considered up to these transformations. The local classification of Dupin surfaces in $\mathbb{R}^{3}$ is well known. Pinkall [8] gave a complete classification up to Lie equivalence for Dupin hypersurfaces $M^{3} \subset \mathbb{R}^{4}$, with three distinct principal curvatures.

Pinkall [7] proved that proper Dupin hypersurfaces $M^{n}$ with $g \geq 3$ that are Lie equivalent to isoparametric hypersurfaces cannot be parametrized by lines of curvature. Niebergall [5,6] and more recently Cecil and Jensen [1] studied proper Dupin hypersurfaces with four distinct principal curvatures and constant Lie curvature (see [4]). Riveros, Rodrigues and Tenenblat [11] studied a class of proper Dupin hypersurfaces $M^{n}$ in $\mathbb{R}^{n+1}$ parametrized by lines of curvature, with $n$ distinct principal curvatures and constant Möbius curvature. They then showed that for $n \geq 3$ the principal curvatures of such hypersurfaces

Received March 18, 2011.

2010 Mathematics Subject Classification. Primary 53A07; Secondary 35N10.

Key words and phrases. lines of curvature, Laplace invariants, Dupin hypersurfaces.

The first author was partially supported by PRONEX-FAPDF and the second author was partially supported by CNPq, CAPES, PROCAD. 
are functions of separated variables and for $n \geq 4$ proper Dupin hypersurfaces $M^{n}$ in $\mathbb{R}^{n+1}$ with $n$ distinct principal curvatures and constant Möbius curvature cannot be parametrized by lines of curvature.

A known result is that in a proper Dupin hypersurface the lines of curvature are circles or straight lines, in both cases the lines of curvature are planar. In this paper, we ask if there exist other hypersurfaces that cannot be parametrized by lines of curvature (aside from the afore-mentioned proper Dupin hypersurfaces $M^{n}$ in $\mathbb{R}^{n+1}$ with $n$ distinct principal curvatures and constant Möbius curvature and those that are Lie equivalent to isoparametric hypersurfaces). The study of the class of hypersurfaces whose lines of curvature are planar and the class of hypersurfaces whose principal curvatures are functions of separated variables is motivated by the results above.

The results presented here are based on the theory of higher-dimensional Laplace invariants introduced by Kamran-Tenenblat [2]-[3]. In [10], Riveros and Tenenblat used the Laplace invariants to obtain a characterization of Dupin hypersurfaces in $\mathbb{R}^{5}$, parametrized by lines of curvature and with four distinct principal curvatures.

This paper is organized as follows. In Section 2, we give some properties of hypersurfaces parametrized by lines of curvature, with distinct principal curvatures. Section 3 is devoted to showing our main results on classes of hypersurfaces $M^{n}$ in $\mathbb{R}^{n+1}$, parametrized by lines of curvature with $n$ distinct principal curvatures and vanishing Laplace invariants.

\section{Preliminaries}

Let $\Omega$ be an open subset of $\mathbb{R}^{n}$ and $x=\left(x_{1}, x_{2}, \ldots, x_{n}\right) \in \Omega$. Let $X: \Omega \subset$ $\mathbb{R}^{n} \rightarrow \mathbb{R}^{n+1}, n \geq 3$, be a hypersurface parametrized by lines of curvature, with distinct principal curvatures $\lambda_{i}, 1 \leq i \leq n$ and let $N: \Omega \subset \mathbb{R}^{n} \rightarrow \mathbb{R}^{n+1}$ be a unit normal vector field of $X$. Then

$$
\begin{aligned}
\left\langle X_{, i}, X_{, j}\right\rangle & =\delta_{i j} g_{i i}, 1 \leq i, j \leq n, \\
N_{, i} & =-\lambda_{i} X_{, i},
\end{aligned}
$$

where the subscript ${ }_{i}$ denotes the derivative with respect to $x_{i}$. Moreover,

$$
\begin{aligned}
& X_{, i j}-\Gamma_{i j}^{i} X_{, i}-\Gamma_{i j}^{j} X_{, j}=0,1 \leq i \neq j \leq n, \\
& \Gamma_{i j}^{i}=\frac{\lambda_{i, j}}{\lambda_{j}-\lambda_{i}}, \quad 1 \leq i \neq j \leq n,
\end{aligned}
$$

where $\Gamma_{i j}^{k}$ are the Christoffel symbols. From (3) we have

$$
\Gamma_{i j, k}^{i}=\Gamma_{i k, j}^{i}, \quad 1 \leq i \neq j \neq k \leq n .
$$

The Christoffel symbols in terms of the metric (1) are given by

$$
\Gamma_{i j}^{k}=0, \quad \Gamma_{i i}^{i}=\frac{g_{i i, i}}{2 g_{i i}}, \quad \Gamma_{i i}^{j}=-\frac{g_{i i, j}}{2 g_{j j}}, \quad \Gamma_{i j}^{i}=\frac{g_{i i, j}}{2 g_{i i}},
$$

where $i, j, k$ are distinct. 
We now consider the higher-dimensional Laplace invariants of the system of equations (3) (see [2]-[3] for the definition of these invariants)

$$
\begin{aligned}
m_{i j} & =-\Gamma_{i j, i}^{i}+\Gamma_{i j}^{i} \Gamma_{i j}^{j}, \\
m_{i j k} & =\Gamma_{i j}^{i}-\Gamma_{k j}^{k}, \quad k \neq i, j, \quad 1 \leq k \leq n .
\end{aligned}
$$

As a consequence of (4) and the un-numbered lemma appearing in [3], we obtain the following identities, valid for distinct $i, j, k, l, 1 \leq i, j, k, l \leq n$ :

$$
\begin{aligned}
m_{i j k}+m_{k j i} & =0, \\
m_{i j k, k}-m_{i j k} m_{j k i}-m_{k j} & =0, \\
m_{i j, k}+m_{i j k} m_{i k}+m_{i k j} m_{i j} & =0, \\
m_{i j k}-m_{i j l}-m_{l j k} & =0, \\
m_{l i k, j}+m_{i j l} m_{k i l}+m_{l j k} m_{k i j} & =0 .
\end{aligned}
$$

From (2) and (6), we obtain

$$
X_{, i i}=\sum_{j} \Gamma_{i i}^{j} X_{, j}-g_{i i} \lambda_{i} N
$$

The Gauss equation for the immersion $X$ is given by

$$
\frac{1}{g_{j j}}\left[\Gamma_{i j, j}^{i}+\Gamma_{i j}^{i}\left(\Gamma_{i j}^{i}-\Gamma_{j j}^{j}\right)\right]+\frac{1}{g_{i i}}\left[\Gamma_{j i, i}^{j}+\Gamma_{j i}^{j}\left(\Gamma_{j i}^{j}-\Gamma_{i i}^{i}\right)\right]+\sum_{k \neq i \neq j} \frac{\Gamma_{i k}^{i} \Gamma_{j k}^{j}}{g_{k k}}+\lambda_{i} \lambda_{j}=0 .
$$

For hypersurfaces with distinct principal curvatures, the Möbius curvature is defined, for distinct $i, j, k$, by

$$
C^{i j k}=\frac{\lambda_{i}-\lambda_{j}}{\lambda_{k}-\lambda_{j}}
$$

Since all $\lambda_{i}$ are distinct we conclude that $C^{i j k} \neq 0$ and $C^{i j k} \neq 1$. Möbius curvatures are invariant under Möbius transformations.

The following result extends Lemma 3.2 in [11] for hypersurfaces parametrized by lines of curvature.

Lemma 2.1. For $n \geq 3$, let $X: \Omega \subset \mathbb{R}^{n} \rightarrow \mathbb{R}^{n+1}$, be a hypersurface parametrized by lines of curvature, with distinct principal curvatures $\lambda_{i}, 1 \leq i \leq n$. Then the Möbius curvatures are constants if and only if

$$
\begin{aligned}
\lambda_{i, i}+\left(\lambda_{i}-\lambda_{k}\right) C^{i j k} m_{j i k} & =0 \text { for distinct } i, j, k, \text { and } \\
m_{j s i}+C^{k s i} C^{i j k} m_{k s j} & =0 \text { for distinct } i, j, k, s .
\end{aligned}
$$

Proof. Let $i, j, k$ and $s$ be distinct. We consider the Möbius curvature $C^{i j k}=$ $\frac{\lambda_{i}-\lambda_{j}}{\lambda_{k}-\lambda_{j}}$. By differentiation we obtain

$$
\begin{aligned}
C_{, i}^{i j k} & =\frac{1}{\lambda_{k}-\lambda_{j}}\left[\lambda_{i, i}+\left(\lambda_{i}-\lambda_{k}\right) C^{i j k} m_{j i k}\right], \\
C_{, j}^{i j k} & =\frac{C^{i k j}}{\lambda_{j}-\lambda_{k}}\left[\lambda_{j, j}+\left(\lambda_{i}-\lambda_{j}\right) C^{j k i} m_{i j k}\right],
\end{aligned}
$$




$$
\begin{aligned}
C_{, k}^{i j k} & =\frac{C^{i j k}}{\lambda_{j}-\lambda_{k}}\left[\lambda_{k, k}+\left(\lambda_{i}-\lambda_{k}\right) C^{k j i} m_{i k j}\right], \\
C_{, s}^{i j k} & =\frac{\lambda_{i}-\lambda_{s}}{\lambda_{k}-\lambda_{j}}\left[m_{j s i}+C^{k s i} C^{i j k} m_{k s j}\right] .
\end{aligned}
$$

Therefore, the Möbius curvature is constant if and only if $C_{i}^{i j k}=0$ and $C_{, s}^{i j k}=$ 0 . These two equations are equivalent to the conditions (12) and (13). Observe that the equations

$$
\begin{aligned}
\lambda_{i, i}+\left(\lambda_{i}-\lambda_{k}\right) C^{i j k} m_{j i k} & =0, \\
\lambda_{j, j}+\left(\lambda_{i}-\lambda_{j}\right) C^{j k i} m_{i j k} & =0, \\
\lambda_{k, k}+\left(\lambda_{i}-\lambda_{k}\right) C^{k j i} m_{i k j} & =0
\end{aligned}
$$

are all equivalent.

Remark 2.2. It follows from Lemma 2.1 that, for Dupin hypersurfaces parametrized by lines of curvature, the condition of having constant Möbius curvature is equivalent to having all higher dimensional Laplace invariants equal to zero.

The following proposition characterizes the hypersurfaces parametrized by planar lines of curvature.

Proposition 2.3. For $n \geq 3$, let $X: \Omega \subset \mathbb{R}^{n} \rightarrow \mathbb{R}^{n+1}$, be a hypersurface parametrized by lines of curvature, with distinct principal curvatures $\lambda_{i}, 1 \leq$ $i \leq n$. The lines of curvature $\alpha_{i}\left(x_{i}\right)=X\left(x_{1}^{0}, x_{2}^{0}, \ldots, x_{i-1}^{0}, x_{i}, x_{i+1}^{0}, \ldots, x_{n}^{0}\right)$, $1 \leq i \leq n$ are planar if and only if

$$
\lambda_{i, i} \Gamma_{i j}^{i}+\lambda_{i} m_{i j}=0,1 \leq i \neq j \leq n .
$$

Proof. We consider the line of curvature $\alpha_{i}\left(x_{i}\right)=X\left(x_{1}^{0}, x_{2}^{0}, \ldots, x_{i-1}^{0}, x_{i}, x_{i+1}^{0}\right.$, $\left.\ldots, x_{n}^{0}\right), 1 \leq i \leq n$ and by differentiation we obtain

$$
\begin{aligned}
\alpha_{i, i} & =X_{, i}, \\
\alpha_{i, i i} & =X_{, i i} .
\end{aligned}
$$

Using (9) in (16) we obtain

$$
\alpha_{i, i i}=\Gamma_{i i}^{i} X_{, i}+\sum_{j \neq i} \Gamma_{i i}^{j} X_{, j}-g_{i i} \lambda_{i} N
$$

Taking the derivative of (17) with respect to $x_{i}$ we have

$\alpha_{i, i i i}=\Gamma_{i i, i}^{i} X_{, i}+\Gamma_{i i}^{i} X_{, i i}+\sum_{j \neq i} \Gamma_{i i, i}^{j} X_{, j}+\sum_{j \neq i} \Gamma_{i i}^{j} X_{, i j}-\left(\lambda_{i, i} g_{i i}+\lambda_{i} g_{i i, i}\right) N-\lambda_{i} g_{i i} N_{, i}$. 
Using (2), (3), (6), (7) and (9) in (18), we have

(19)

$$
\begin{aligned}
\alpha_{i, i i i}= & {\left[\Gamma_{i i, i}^{i}+\left(\Gamma_{i i}^{i}\right)^{2}+\sum_{j \neq i} \Gamma_{i i}^{j} \Gamma_{i j}^{i}-\left(\lambda_{i}\right)^{2} g_{i i}\right] X_{i}+\sum_{j \neq i} \frac{g_{i i}}{g_{j j}}\left(m_{i j}-3 \Gamma_{i i}^{i} \Gamma_{i j}^{i}\right) X_{, j} } \\
& -g_{i i}\left[3 \lambda_{i} \Gamma_{i i}^{i}+\lambda_{i, i}\right] N .
\end{aligned}
$$

The lines of curvature $\alpha_{i}$ are planar if and only if $\alpha_{i, i}, \alpha_{i, i i}$ and $\alpha_{i, i i i}$ are linearly dependent, that is, there exist scalars $a, b, c$ not all zero, such that

$$
a \alpha_{i, i}+b \alpha_{i, i i}+c \alpha_{i, i i i}=0 \text {. }
$$

Substituting (15), (17) and (19) in (20) we have

$$
\begin{aligned}
& \left\{a+b \Gamma_{i i}^{i}+c\left[\Gamma_{i i, i}^{i}+\left(\Gamma_{i i}^{i}\right)^{2}+\sum_{j \neq i} \Gamma_{i i}^{j} \Gamma_{i j}^{i}-\left(\lambda_{i}\right)^{2} g_{i i}\right]\right\} X_{, i} \\
& +\sum_{j \neq i}\left[b \Gamma_{i i}^{j}+\frac{c g_{i i}}{g_{j j}}\left(m_{i j}-3 \Gamma_{i i}^{i} \Gamma_{i j}^{i}\right)\right] X_{, j}-g_{i i}\left[b \lambda_{i}+c\left(3 \lambda_{i} \Gamma_{i i}^{i}+\lambda_{i, i}\right)\right] N=0 .
\end{aligned}
$$

The equation (21) is equivalent to the system

$$
\begin{aligned}
a+b \Gamma_{i i}^{i}+c\left[\Gamma_{i i, i}^{i}+\left(\Gamma_{i i}^{i}\right)^{2}+\sum_{j \neq i} \Gamma_{i i}^{j} \Gamma_{i j}^{i}-\left(\lambda_{i}\right)^{2} g_{i i}\right] & =0, \\
-b \Gamma_{i j}^{i}+c\left(m_{i j}-3 \Gamma_{i i}^{i} \Gamma_{i j}^{i}\right) & =0, j \neq i \\
b \lambda_{i}+c\left(3 \lambda_{i} \Gamma_{i i}^{i}+\lambda_{i, i}\right) & =0 .
\end{aligned}
$$

The system (22) has a nontrivial solution $a, b, c$, if for distinct $i, j, k$

$$
\begin{aligned}
\lambda_{i, i} \Gamma_{i j}^{i}+\lambda_{i} m_{i j} & =0, \text { and } \\
\Gamma_{i j}^{i} m_{i k}-\Gamma_{i k}^{i} m_{i j} & =0 .
\end{aligned}
$$

We observe that (23) implies (24), which is equivalent to (14).

Conversely, if $\lambda_{i, i} \Gamma_{i j}^{i}+\lambda_{i} m_{i j}=0$ we obtain the following facts:

If $\lambda_{i}=0$, from (17) and (19) it follows $\alpha_{i, i i}=\Gamma_{i i}^{i} \alpha_{, i}, \alpha_{i, i i i}=\left[\Gamma_{i i, i}^{i}+\left(\Gamma_{i i}^{i}\right)^{2}\right] \alpha_{, i}$. If $\lambda_{i} \neq 0$, then

$$
\alpha_{i, i i i}=\left[\Gamma_{i i, i}^{i}-2\left(\Gamma_{i i}^{i}\right)^{2}+\sum_{j \neq i} \Gamma_{i i}^{j} \Gamma_{i j}^{i}-\left(\lambda_{i}\right)^{2} g_{i i}-\frac{\lambda_{i, i}}{\lambda_{i}} \Gamma_{i i}^{i}\right] \alpha_{i, i}+\left[\frac{\lambda_{i, i}}{\lambda_{i}}+3 \Gamma_{i i}^{i}\right] \alpha_{i, i i} .
$$

Therefore, in both cases $\alpha_{i, i}, \alpha_{i, i i}$ and $\alpha_{i, i i i}$ are linearly dependent. Hence, the lines of curvature $\alpha_{i}$ are planar.

We further need the following result. 
Lemma 2.4. (i) For $n \geq 4$, there does not exist a hypersurface $M^{n}$ in $\mathbb{R}^{n+1}$, parametrized by lines of curvature, with $n$ distinct principal curvatures $\lambda_{i}$, $m_{k j i}=0, \forall 1 \leq i \neq j \neq k \leq n$ and

$$
\lambda_{i, i} \Gamma_{i j}^{i}=0, \quad 1 \leq i \neq j \leq n,
$$

(ii) Up to Möbius transformations, there is a unique proper hypersurface $M^{3}$, immersed in $\mathbb{R}^{4}$, parametrized by lines of curvature, with three distinct principal curvatures, $m_{k j i}=0$ and $\lambda_{i, i} \Gamma_{i j}^{i}=0,1 \leq i \neq j \leq 3$.

Proof. (i) Assume that $n \geq 4$, and that there exists such a hypersurface $M^{n}$ in $\mathbb{R}^{n+1}$, satisfying the hypotheses of the lemma and let $X\left(x_{1}, \ldots, x_{n}\right)$ be a local parametrization of $M^{n}$ by lines of curvature. Assuming that $\lambda_{i, i} \neq 0, \forall i \neq j$, we have (from (25))

$$
\Gamma_{i j}^{i}=0, \forall 1 \leq i \neq j \leq n .
$$

Using (26) in the Gauss equation (10), we obtain

$\Gamma_{j i, i}^{j}+\Gamma_{j i}^{j}\left(\Gamma_{j i}^{j}-\Gamma_{i i}^{i}\right)+\lambda_{i} \lambda_{j} g_{i i}=0, \quad \Gamma_{s i, i}^{s}+\Gamma_{s i}^{s}\left(\Gamma_{s i}^{s}-\Gamma_{i i}^{i}\right)+\lambda_{i} \lambda_{s} g_{i i}=0, i \neq j \neq s$.

From (27) and the fact that $m_{j i s}=0$ we obtain

$$
g_{i i} \lambda_{i}\left(\lambda_{j}-\lambda_{s}\right)=0 .
$$

From (28) we get $\lambda_{i}=0$, which is a contradiction. Therefore, $\lambda_{i, i}=0, \forall i$ and $M^{n}$ is Dupin. Now Remark 2.2 implies that $M^{n}$ has constant Möbius curvature, which is a contradiction, since we know that there are no Dupin hypersurfaces parametrized by lines of curvature with constant Möbius curvature (see Theorem A in [11]).

(ii) Using a similar argument, we may show that $M^{3}$ is Dupin. Remark 2.2 then implies that $M^{3}$ has constant Möbius curvature. The result then follows from [11] (see Theorem B).

Remark 2.5. From the second equation of (8), we obtain that if $m_{k j i}=0, \forall i \neq$ $j \neq k$ then $m_{i j}=0, \forall i \neq j$.

\section{Main results}

The following theorem characterizes a class of hypersurfaces $M^{3} \subset \mathbb{R}^{4}$, with three distinct principal curvatures at each point, parametrized by planar lines of curvature and vanishing Laplace invariants, in terms of the Dupin hypersurfaces with constant Möbius curvature.

Theorem 3.1. Let $M^{3} \subset \mathbb{R}^{4}$, be a hypersurface parametrized by lines of curvature, with three distinct principal curvatures $\lambda_{i} . M^{3}$ has planar lines of curvature and $m_{k j i}=0, \forall 1 \leq i \neq j \neq k \leq 3$ if and only if $M^{3}$ is Dupin with constant Möbius curvature. 
Proof. Let $X\left(x_{1}, x_{2}, x_{3}\right)$ be a local parametrization of $M^{3}$ by lines of curvature. If the lines of curvature of $M^{3}$ are planar, it follow from Proposition 2.3 that

$$
\lambda_{i, i} \Gamma_{i j}^{i}+\lambda_{i} m_{i j}=0,1 \leq i \neq j \leq 3 .
$$

Using Remark 2.5, (29) reduces to

$$
\lambda_{i, i} \Gamma_{i j}^{i}=0,1 \leq i \neq j \leq 3 .
$$

It follows from Lemma 2.4 (item (ii)) and Remark 2.2 that $M^{3}$ is Dupin with constant Möbius curvature.

Conversely, if $M^{3}$ is Dupin with constant Möbius curvature then all higher dimensional Laplace invariants are equal to zero, i.e., $m_{i j}=m_{k j i}=0$ for all distinct $i, j, k \in\{1,2,3\}$ (recall Remark 2.2). Therefore (14) is satisfied by the lines of curvature of $M^{3}$, i.e., the lines of curvature are planar.

Theorem 3.2. For $n \geq 4$, there does not exist a hypersurface $M^{n}$ in $\mathbb{R}^{n+1}$, parametrized by planar lines of curvature, with $n$ distinct principal curvatures $\lambda_{i}$ and $m_{k j i}=0, \forall 1 \leq i \neq j \neq k \leq n$.

Proof. Assume that $n \geq 4$, and that there exists such a hypersurface $M^{n}$ in $\mathbb{R}^{n+1}$. Let $X\left(x_{1}, \ldots, x_{n}\right)$ be a local parametrization of $M^{n}$ by lines of curvature. Since the lines of curvature of $M^{n}$ are planar, it follows from Proposition 2.3 that $\lambda_{i, i} \Gamma_{i j}^{i}+\lambda_{i} m_{i j}=0,1 \leq i \neq j \leq n$. From Remark 2.5 this equation reduces to $\lambda_{i, i} \Gamma_{i j}^{i}=0$, which contradicts item (i) of Lemma 2.4.

Theorem 3.3. Let $M^{3} \subset \mathbb{R}^{4}$, be a hypersurface parametrized by lines of curvature, with three distinct principal curvatures $\lambda_{i}$. The hypersurface $M^{3}$ is Dupin with constant Möbius curvature if and only if the following conditions hold:

(i) $m_{k j i}=0$ for all distinct $i, j, k \in\{1,2,3\}$ and

(ii) there exists functions of separated variables $\left\{f_{i r}\right\}_{i, r=1}^{3}$ so that $\lambda_{i}=$ $\sum_{r=1}^{3} f_{i r}\left(x_{r}\right)$.

Proof. We observe that (4) and (7) yield

$$
\lambda_{i, j i}=\left(\lambda_{i}-\lambda_{j}\right) m_{i j}-\lambda_{i, i} \Gamma_{i j}^{i}, 1 \leq i \neq j \leq n .
$$

If $M^{3}$ is Dupin with constant Möbius curvature, then $m_{i j k}=m_{i j}=0,1 \leq i \neq$ $j \neq k \leq 3$ and therefore

$$
\lambda_{i, i j}=0,1 \leq i \neq j \leq 3 .
$$

Moreover, using (4), (5) and the fact that $m_{i j k}=0$, we obtain

$$
\lambda_{i, j k}=0,1 \leq i \neq j \neq k \leq 3 .
$$

It follow from (32) and (33) that $\lambda_{i}=\sum_{r=1}^{3} f_{i r}\left(x_{r}\right)$.

Conversely, by hypothesis, $\lambda_{i, j i}=0, \forall 1 \leq i \neq j \leq 3$. Using Remark 2.5, (31) reduces to

$$
\lambda_{i, i} \Gamma_{i j}^{i}=0
$$


It follows from Lemma 2.4 (item (ii)) and Remark 2.2 that $M^{3}$ has constant Möbius curvature.

Theorem 3.4. (i) For $n \geq 4$, there does not exist a hypersurface $M^{n}$ in $\mathbb{R}^{n+1}$, parametrized by lines of curvature, with $n$ distinct principal curvatures $\lambda_{i}$, satisfying $m_{k j i}=0, \forall 1 \leq i \neq j \neq k \leq n$ and $\lambda_{i}=\sum_{r=1}^{n} g_{i r}\left(x_{r}\right)$.

(ii) Up to Möbius transformations, there is a unique proper hypersurface $M^{3}$, immersed in $\mathbb{R}^{4}$, parametrized by lines of curvature, with three distinct principal curvatures, $m_{k j i}=0$ and $\lambda_{i}=\sum_{r=1}^{3} g_{i r}\left(x_{r}\right)$.

Proof. (i) Assume that $n \geq 4$, and that there exists such a hypersurface $M^{n}$ in $\mathbb{R}^{n+1}$. Let $X\left(x_{1}, \ldots, x_{n}\right)$ be a parametrization of $M^{n}$ by lines of curvature. By hypothesis, $\lambda_{i, j i}=0, \forall 1 \leq i \neq j \leq n$. Using Remark 2.5, (31) reduces to

$$
\lambda_{i, i} \Gamma_{i j}^{i}=0
$$

which contradicts the Lemma 2.4 (item (i)).

(ii) Theorem 3.3 implies that $M^{3}$ is Dupin with constant Möbius curvature. The result then follows from [11] (see Theorem B).

\section{References}

[1] T. E. Cecil and G. Jensen, Dupin hypersurfaces with four principal curvatures, Geom. Dedicata 79 (2000), no. 1, 1-49.

[2] N. Kamran and K. Tenenblat, Laplace transformation in higher dimensions, Duke Math. J. 84 (1996), no. 1, 237-266.

[3] _ Periodic systems for the higher-dimensional Laplace transformation, Discrete Contin. Dynam. Systems 4 (1998), no. 2, 359-378.

[4] R. Miyaoka, Dupin hypersurfaces and a Lie invariant, Kodai Math. J. 12 (1989), no. 2, $228-256$.

[5] R. Niebergall, Dupin hypersurfaces in $\mathbb{R}^{5}$, Part I, Geom. Dedicata 40 (1991), no. 1, $1-22$.

[6] _ Dupin hypersurfaces in $\mathbb{R}^{5}$, Part II, Geom. Dedicata 41 (1992), no. 1, 5-38.

[7] U. Pinkall, Dupin'sche Hyperflachen, Dissertation, Univ. Freiburg, 1981.

[8] _ Dupin'sche hyperflachen in $E^{4}$, Manuscripta Math. 51 (1985), 89-119.

[9] _ Dupin hypersurfaces, Math. Ann. 270 (1985), 427-440.

[10] C. M. C. Riveros and K. Tenenblat, Dupin hypersurfaces in $\mathbb{R}^{5}$, Canad. J. Math. 57 (2005), no. 6, 1291-1313.

[11] C. M. C. Riveros, K. Tenenblat, and L. A. Rodrigues, On Dupin hypersurfaces with constant Möbius curvature, Pacific J. Math. 236 (2008), no. 1, 89-103.

Carlos M. C. Riveros

Departamento de Matemática

Universidade DE BRasília

Brasília 70910-900, BRAZIL

E-mail address: carlos@mat.unb.br

Armando M. V. Corro

Instituto de Matemática e Estatística

Universidade Federal de GoiÂS

GoiÂNIA 74001-970, BRAZIL

E-mail address: corro@mat.ufg.br 